\title{
A LEI DO SANEAMENTO BÁSICO E SEU IMPACTO NOS ÍNDICES DE ACESSO AOS SERVIÇOS DE SANEAMENTO BÁSICO ${ }^{1,2}$
}

\author{
Damares Lopes Afonso 3 \\ Eduardo Simões de Almeida ${ }^{4}$
}

\begin{abstract}
O Brasil ainda enfrenta desafios quanto ao oferecimento dos serviços de saneamento básico à população. Em busca da universalização desses serviços, o governo federal promulgou a Lei no 11.445, a chamada Lei do Saneamento Básico de 2007. Dentre as diretrizes fundamentadas pela lei, encontra-se a elaboração dos planos municipais de saneamento básico (PMSB) por parte de todos os municípios brasileiros. 0 presente trabalho propõe avaliar o impacto da Lei do Saneamento Básico sobre os índices de acesso aos serviços de saneamento básico relacionados ao abastecimento de água, à coleta de lixo e ao esgotamento sanitário dos municípios. Utilizase a combinação do modelo de diferenças em diferenças (DD) com pareamento por escore de propensão, tendo como tratamento o fato de os municípios terem elaborado seus PMSB, ou seja, estarem em conformidade com a lei. Os resultados indicam que a aplicação da lei teve impacto positivo e significativo nos índices de acesso aos serviços de coleta de lixo e esgotamento sanitário.
\end{abstract}

Palavras-chave: Lei do Saneamento Básico; planos municipais de saneamento básico; diferenças em diferenças; pareamento por escore de propensão.

\section{THE LAW OF BASIC SANITATION AND ITS IMPACT ON THE RATES OF ACCESS TO BASIC SANITATION SERVICES}

Brazil still faces challenges regarding the provision of basic sanitation services to the population. In pursuit of the universalization of these services, the federal government enacted Law no. 11,445, the Basic Sanitation Law of 2007. Among the guidelines presents on the Law, is the elaboration of Municipal Plans of Basic Sanitation (PMSB) by all the Brazilian municipalities. The present work proposes to evaluate the impact of the Basic Sanitation Law on the indices of access to basic sanitation services related to water supply, garbage collection and sanitary sewage of municipalities. The combine the Differences in Differences with propensity score matching model is used, having as treatment the fact that the municipalities have elaborated their PSMB, having as treatment the fact that the municipalities have elaborated their PSMB, that is, they was in compliance with the Law. The results indicate that the application of the Law had a positive and significant impact on the rates of access to garbage and sewage collection services.

Keywords: Basic Sanitation Law; municipal plans of basic sanitation; difference in differences; propensity score matching.

1. DOI: http://dx.doi.org/10.38116/ppp56art5

2. A autora agradece o financiamento da Coordenação de Aperfeiçoamento de Pessoal de Nível Superior (Capes) e da Fundação de Amparo à Pesquisa do Estado de Minas Gerais (Fapemig). 0 autor agradece o financiamento do Conselho Nacional de Desenvolvimento Científico e Tecnológico (CNPq) e da Fapemig.

3. Doutoranda em economia na Universidade Federal de Juiz de Fora (UFJF).E-mail: <damaresalopes@gmail.com>.

4. Professor da UFJF e pesquisador do CNPq. E-mail: <eduardo.almeida@ufjf.edu.br>. 


\title{
LA LEY DEL SANEAMIENTO BÁSICO Y SU IMPACTO EN LOS ÍNDICES DE ACCESO A LOS SERVICIOS DE SANEAMIENTO BÁSICO
}

\begin{abstract}
Brasil todavía enfrenta desafíos en cuanto al ofrecimiento de los servicios de saneamiento básico a la población. En la búsqueda de la universalización de estos servicios, el gobierno federal promulgó la Ley no 11.445, la llamada Ley del Saneamiento Básico de 2007. Entre las directrices fundamentadas por la Ley, se encuentra la elaboración de los Planes Municipales de Saneamiento Básico (PMSB) por parte de todos los municipios brasileños. El presente trabajo propone evaluar el impacto de la Ley del Saneamiento Básico sobre los índices de acceso a los servicios de saneamiento básico relacionados al abastecimiento de agua, recolección de basura y agotamiento sanitario de los municipios. Se utiliza la combinación del modelo de Diferencias en Diferencias con pareamiento por puntuación de propensión, teniendo como tratamiento el hecho de que los municipios hayan elaborado sus PMSB, o sea, estar en conformidad con la Ley. Los resultados indican que la aplicación de la Ley tuvo un impacto positivo y significativo en las tasas de acceso a los servicios de recolección de basura y recolección de aguas residuales.
\end{abstract}

Palabras clave: Ley del Saneamiento Básico; Planes Municipales de Saneamiento Básico (PMSB); Diferencias en Diferencias; Pareamiento por Puntaje de Propensión.

\section{LA LOI DE L'ASSAINISSEMENT DE BASE ET SON IMPACT SUR LES TAUX D'ACCÈS AUX SERVICES D'ASSAINISSEMENT DE BASE}

Le Brésil est toujours confronté à des défis concernant la fourniture de services d'assainissement de base à la population. Pour promouvoir ces services universels, le gouvernement fédéral a adopté la loi n ${ }^{\circ} 11445$, appelé la Loi sur l'hygiène de base des 2007. Parmi les lignes directrices fondées par la loi, est la rédaction des plans d'assainissement de base municipaux (PMSB) par tous les les municipalités brésiliennes. Cet article propose d'évaluer l'impact de la Loi sur l'hygiène de base sur les taux d'accès aux services d'assainissement de base liés à l'approvisionnement en eau, la collecte des ordures et des eaux usées des municipalités. En utilisant la combinaison de la méthode Difference in Differences avec la correspondance par score de propension, le traitement du fait que les municipalités ont préparé leur PMSB, à savoir se conformer à la loi. Les résultats indiquent que I'application de la loi a eu une incidence positive et significative sur les taux d'accès aux services de collecte des ordures ménagères et des eaux usées.

Mots-clés: Loi fondamentale de l'assainissement; les plans d'assainissement municipaux (PMSB); Différences en Différences; Appariement par Score de Propension.

\section{INTRODUÇÃO}

Segundo a Organização Mundial da Saúde - OMS (1950), entende-se por saneamento básico o controle de todos os fatores do meio físico que exercem ou podem exercer efeitos nocivos sobre o bem-estar físico, mental e social. Dessa forma, a relação entre saúde e saneamento se dá por este último higienizar o ambiente, atuando na prevenção de doenças que podem proliferar-se com dejetos, lixo, contaminação da água, entre outros (Heller, 1998).

Apesar dos avanços no acesso aos serviços de saneamento básico obtidos no decorrer dos anos 2000 em todo o território brasileiro, permanece a existência de deficit no acesso e heterogeneidade na distribuição desses serviços no país, com 
concentração desses na região Centro-Sul ${ }^{5}$ e deficit principalmente nas regióes Norte e Nordeste, além das diferenças existentes entre o atendimento das populaçôes urbanas e rurais (Saiani e Toneto Júnior, 2010; IBGE, 2011; Galvão, 2013).

A concentração desses serviços em regióes economicamente mais desenvolvidas e urbanizadas está relacionada às diferenças no perfil de renda dos municípios brasileiros, em razão da capacidade de pagamento das tarifas de seus consumidores, e à existência de economias de escala, tendo em vista os altos custos de prestação desses serviços (Saiani, 2006).

Buscando a universalização e a regulação dos serviços de saneamento básico, o governo federal proclamou a Lei no 11.445 , em 5 de janeiro de 2007, Lei de Saneamento Básico, que expóe as diretrizes necessárias à prestação do saneamento básico no território brasileiro. Esta lei considera como serviços de saneamento básico estas atividades: i) abastecimento de água; ii) esgotamento sanitário; iii) limpeza urbana e manejo de resíduos; e iv) drenagem e manejo de águas pluviais, limpeza e fiscalização preventiva das respectivas redes urbanas (Brasil, 2007).

A Lei no 11.445/2007 outorga os municípios como entes responsáveis pela prestação dos serviços de saneamento básico, podendo estes contratarem empresas prestadoras, fazer parcerias municipais, entre outros. Porém, a prestação desses serviços deve ser planejada pelos próprios municípios, mediante a elaboração do plano municipal de saneamento básico. Os PMSB estabelecem as diretrizes municipais na área de saneamento e devem estar em consonância com as diretrizes nacionais estabelecidas pela Lei no 11.445/2007 e pelo Decreto nº 7.217/2010 (Brasil, 2010).

De acordo com o texto da Lei no 11.445/2007, o PMSB deve conter no mínimo: i) diagnóstico da situação e de seus impactos nas condiçóes de vida; ii) objetivos e metas de curto, médio e longo prazos para a universalização; iii) programas, projetos e açóes necessárias para atingir os objetivos e as metas; iv) açôes para emergências e contingências; e v) mecanismos e procedimentos para a avaliação sistemática da eficiência e eficácia das açóes programadas.

Com o Decreto no 7.217/2010, a existência do PMSB também passaria a ser condição para os municípios terem acesso aos recursos públicos destinados ao saneamento básico a partir de 2014. No entanto, esta cláusula foi modificada três vezes até 2017 - em 2014 o Decreto no 8.211/2014 alterou a data do condicionamento dos recursos para 31 de dezembro de 2015; o Decreto no 8.629/2015 alterou esta data para 31 de dezembro de 2017; o Decreto no 9.254/2017, por sua vez, alterou novamente a data para 31 de dezembro de 2019.

5. Corresponde às regiões Sul e Sudeste, com exceção da região norte de Minas Gerais. Além dos estados de Mato Grosso do Sul, Goiás, sul do Tocantins, sudeste de Mato Grosso e o Distrito Federal. 
Um dos motivos das alterações nos decretos deve-se ao baixo número de municípios que concluíram a elaboração de seus PMSB. Até 2016 apenas 30\% do total dos municípios brasileiros haviam elaborado de fato seus planos segundo dados do documento Panorama dos planos municipais de saneamento básico, publicado em 2017 pela Secretaria Nacional de Saneamento Ambiental, ligada ao Ministério das Cidades.

Diante desse cenário, o objetivo deste trabalho é avaliar se a Lei do Saneamento Básico de 2007 teve impacto sobre os índices de acesso aos serviços de saneamento referentes ao abastecimento de água, à coleta de lixo e ao esgotamento sanitário. Por abastecimento de água entende-se o fornecimento de água potável por rede de distribuição, poço, nascente ou cisterna, com canalização interna. A coleta de lixo está vinculada a limpeza urbana e manejo de resíduos, e o esgotamento sanitário compreende a coleta de esgoto por rede ou o uso de fossa séptica (Brasil, 2013).

Para avaliar as diferenças entre os grupos de tratamento e controle, utiliza-se a combinação da metodologia diferenças em diferenças com pareamento por escore de propensão. Como a Lei no 11.445/2007 dirige-se a todos os municípios brasileiros, para calcular o seu impacto utilizam-se como grupo de tratamento os municípios que de fato a cumpriram, ou seja, aqueles que efetivamente elaboraram seus PMSB. Já o grupo de controle, não afetado pela lei, refere-se aos municípios que não haviam elaborado seu plano, de acordo com o documento Panorama dos planos municipais de saneamento básico.

O pareamento por escore de propensão é utilizado para contornar o problema do viés de seleção, que advém do fato da efetiva participação dos municípios na lei, ou seja, a elaboração de seus PMSB não ser aleatória, estando vinculada a atributos próprios dos municípios, como seu índice de desenvolvimento, e o fato de estes pertencerem à regiáo geoeconômica Centro-Sul do Brasil.

O período de análise compreende os anos de 2005, período anterior à aplicação da Lei do Saneamento Básico de 2007, e 2015, período posterior a essa lei. Os resultados apontam um impacto positivo e significativo da lei sobre os índices de coleta de lixo e esgotamento sanitário dos municípios brasileiros e não significativo para o índice de acesso aos serviços de abastecimento de água.

Este trabalho contribui com uma avaliação do impacto da Lei do Saneamento Básico de 2007 nos municípios brasileiros. Até o momento desta pesquisa, não se têm evidências de estudos empíricos que avaliaram o impacto dessa lei no Brasil, uma das razóes é por esta ser uma política recente, além do fato de existirem poucos dados a respeito. Dessa forma, pretende-se preencher essa lacuna. 
Além desta introdução, o artigo está organizado em mais quatro seçóes. A próxima seção faz uma contextualização geral a respeito dos serviços de saneamento básico no Brasil. Em seguida, aborda-se a estratégia empírica adotada e descreve-se a base de dados utilizada. Por fim, apresentam-se os resultados e a conclusão, respectivamente.

\section{OS SERVIÇOS DE SANEAMENTO BÁSICO NO BRASIL}

Os investimentos em saneamento no Brasil ocorreram de forma descontínua ao longo do tempo, além de terem sido realizados de maneira desarticulada entre as esferas de governo e serem predominantemente financiados pelo setor público (Leoneti, Prado e Oliveira, 2011).

Nas décadas de 1970-1980, institui-se o Plano Nacional de Saneamento (Planasa), que propôs investimentos na área de saneamento básico, sendo atendidos principalmente o abastecimento de água e o esgotamento sanitário (Pires, 1979). O Planasa geria-se principalmente com recursos públicos, e foi deixado em função da crise econômica dos anos 1980 . Na década de 1990 a política de saneamento básico nacional orientou-se com base nos ideais liberais, atendendo medidas de desregulamentação do setor, abertura à iniciativa privada, além da privatização de algumas prestadoras públicas (Maso, 2012).

Passados alguns anos sem amplas articulaçóes na esfera do planejamento em saneamento básico no Brasil, em 5 de janeiro de 2007, foi promulgada a Lei $\mathrm{n}$ ำ11.445, a qual assume a universalizaçáo do saneamento básico como um compromisso de toda a sociedade brasileira e atua como um esforço de reforma institucional que envolve governos, prestadores de serviços, indústria, agentes financeiros e a sociedade, por meio de suas organizaçôes e dos canais de participação (Brasil, 2009).

O Conselho das Cidades iniciou em 2008 a elaboração do Plano Nacional de Saneamento Básico (Plansab), articulado mediante a Lei no 11.445/2007 em três etapas de planejamento: i) formulação do "Pacto pelo Saneamento Básico: mais saúde, qualidade de vida e cidadania”, em 2008; ii) elaboração, em 2009 e 2010, do estudo denominado Panorama do saneamento básico no Brasil, que desencadeou a versão preliminar do Plansab; iii) "Consulta pública", que submeteu a versão preliminar do Plansab à sociedade (Brasil, 2013). O Plansab deveria ser revisado a cada quatro anos, estabelecendo novas metas e programaçáo para o alcance destas.

Ainda em conformidade com a Lei no $11.445 / 2007$, os municípios brasileiros também deveriam elaborar seus PMSB. Esses planos podem ser considerados como um diagnóstico completo de avaliação da situação e do planejamento dos serviços de saneamento básico e aspectos relacionados a estes, necessitando de uma equipe interdisciplinar qualificada para sua elaboração. 
Os municípios brasileiros encontram dificuldades na construção de seus PMSB, e os principais desafios referem-se à falta de recursos financeiros, falta de profissionais qualificados e de capacidade técnica municipal para a sua elaboração (Lisboa, Heller e Silveira, 2013). Além disso, mesmo na presença desses recursos, os municípios podem enfrentar outros impasses políticos na uniáo de forças para a elaboração de "bons" planos (Pereira e Heller, 2015).

De acordo com o Panorama dos planos municipais de saneamento básico, apenas $30 \%$ dos municípios brasileiros haviam elaborado seus PMSB. O panorama é uma síntese dos levantamentos realizados por fontes governamentais, como o Instituto Brasileiro de Geografia e Estatística (IBGE), o Sistema Nacional de Informaçóes em Saneamento (SNIS), e fontes não governamentais, oriundas de estudos e pesquisas realizados por entidades do setor de saneamento básico no Brasil, abrangendo o período de 2011 a 2016 (Brasil, 2017).

O panorama foi construído em três etapas: i) consulta e sistematização dos dados secundários existentes sobre o assunto; ii) aplicação de um questionário, enviado aos municípios brasileiros; e iii) consolidação dos resultados oriundos das diversas fontes adotadas em um banco de dados (Brasil, 2017).

$\mathrm{O}$ banco de dados do panorama informa quantos e quais municípios declararam que haviam elaborado seus PMSB ou estavam com estes em fase de elaboração entre os anos de 2011 e 2016. A publicação também identificou municípios que apresentavam inconsistências de informação - por exemplo, municípios que em um dos anos declararam que haviam elaborado o PMSB, mas que em anos mais recentes das pesquisas afirmaram que este estava em fase de elaboração.

Além disso, há municípios cujas informaçôes não constam no panorama, uma vez que não responderam aos questionários e às pesquisas analisadas por este. Nesse caso não se pode atribuir que não tenham o PMSB, apenas que não fazem parte da amostra apurada no panorama (Brasil, 2017).

A tabela 1 traz uma síntese ${ }^{6}$ dos municípios brasileiros, agregados por região, que, segundo o panorama, apresentavam informaçóes inconsistentes nas pesquisas com relação à elaboração de seus PMSB, municípios que de fato haviam elaborado seus PMSB ou estavam em fase de elaboração, além dos municípios sem informação no panorama.

6. Quatro municípios com informações contidas no panorama, mas que foram criados posteriormente ao ano de 2005 (ano de controle), foram deixados de fora da análise. São esses: Balneário Rincão (SC), Paraíso das Águas (MS), Pescaria Brava (SC) e Nazária (PI). 
TABELA 1

Panorama dos planos municipais de saneamento básico no Brasil

\begin{tabular}{lccccc}
\hline Situação & Inconsistência & Possui plano & Plano em elaboração & Sem informação & Total \\
\hline Norte & 8 & 99 & 182 & 162 & 451 \\
Nordeste & 8 & 184 & 804 & 798 & 1.794 \\
Centro-0este & 13 & 54 & 294 & 105 & 466 \\
Sudeste & 70 & 662 & 561 & 375 & 1.668 \\
Sul & 21 & 691 & 248 & 231 & $\mathbf{1 . 1 9 1}$ \\
Brasil & 120 & 1.690 & $\mathbf{2 . 0 8 9}$ & $\mathbf{1 . 6 7 1}$ & $\mathbf{5 . 5 7 0}$ \\
\hline
\end{tabular}

Fonte: Panorama dos Planos Municipais de Saneamento Básico.

Elaboração dos autores.

No que diz respeito aos investimentos em saneamento básico no Brasil, é possível observar seu aumento no período posterior à Lei do Saneamento Básico de 2007. Os avanços propostos pela lei foram em parte viabilizados pelas obras do Programa de Aceleraçáo do Crescimento (PAC). Entre 2003 e 2006 a média de desembolso federal em saneamento foi de R \$ 1,57 bilhão ao ano, mas esse aporte anual passou para $\mathrm{R} \$ 7,14$ bilhóes entre 2007 e 2015, período compreendido pelos PACs 1 e 2 (Brasil, 2016).

Entretanto, esse aumento ainda é insuficiente para a universalização dos serviços em todo o território brasileiro. Estima-se que seriam necessários ao menos sete PACs consecutivos para que todos os brasileiros tivessem acesso aos serviços de saneamento básico (Carlos, 2013).

Além disso, os investimentos em saneamento, bem como outros investimentos em infraestrutura no Brasil, também enfrentam desafios, como a falta de coordenaçáo das açóes da administração pública entre os níveis municipal, estadual e federal, além de ineficiências ligadas à gestão dos recursos e dos processos (Frischtak, 2008; Trata Brasil, 2010; Marcelini, 2016).

Apesar do aumento dos investimentos entre 2007-2015, o caráter heterogêneo na distribuição dos serviços de saneamento no Brasil perdura, tendo as regióes Norte e Nordeste maior deficiência na distribuiçẫo desses serviços comparativamente à regiāo Centro-Sul do país (Venson, Rodrigues e Camara, 2015; Afonso e Perobelli, 2018).

A figura 1 mostra a distribuição espacial dos índices de acesso aos serviços públicos de saneamento básico nos municípios brasileiros - são considerados os serviços ligados ao abastecimento de água, à coleta de lixo e ao esgotamento sanitário, previstos pela Lei no $11.445 / 2007$. 
Os dados ${ }^{7}$ são provenientes do Sistema de Informações Sobre a Atenção Básica (SIAB), disponíveis na plataforma Datasus, ${ }^{8}$ e informam o percentual de famílias cadastradas na atenção básica com acesso aos serviços de saneamento básico analisados nos anos de 2005 e 2015.

FIGURA 1

Distribuição do acesso aos serviços de abastecimento de água, coleta de lixo e esgotamento sanitário nos municípios brasileiros (2005 e 2015)

$1 \mathrm{~A}$ - Proporção de famílias com abastecimento de águas por rede geral, poço ou nascente
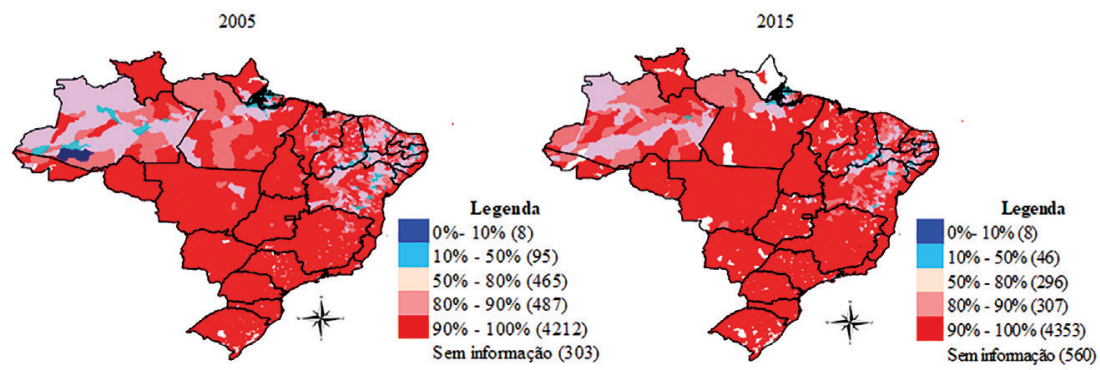

1B - Proporção de famílias com lixo coletado 2005
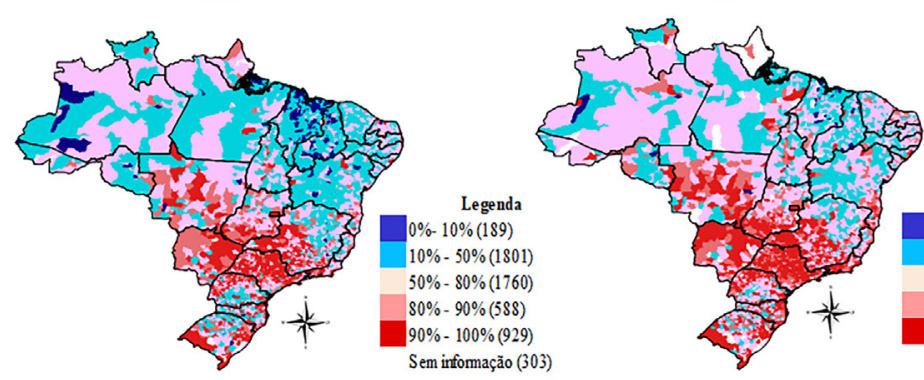

Legenda

$0 \%-10 \%(40)$

$10 \%-50 \%(1049)$

$50 \%-80 \%(1883)$

$80 \%-90 \%(776)$

$90 \%$ - 100\% (1262)

Sem informação (560)

1C - Proporção de famílias com rede de esgoto ou fossa séptica 2005

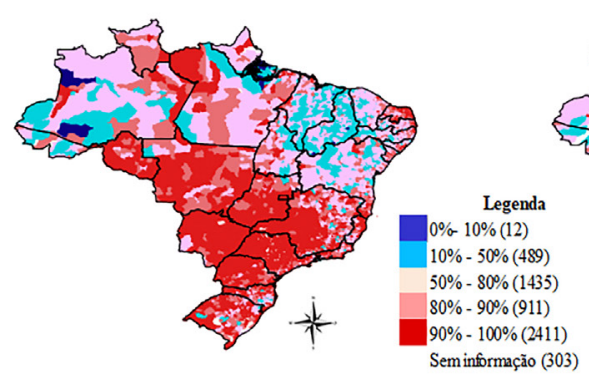

2015

Fonte: ArcView 3.2, com base nos dados do SIAB.

Obs.: Figura reproduzida em baixa resolução e cujos leiaute e textos não puderam ser padronizados e revisados em virtude das condições técnicas dos originais (nota do Editorial). 
Pela figura 1 é possível observar que o deficit na cobertura dos serviços de saneamento ligados à coleta de lixo e ao esgotamento sanitário são maiores comparativamente aos serviços de abastecimento de água. Nota-se que o acesso das famílias a esses serviços aumentou em 2015 comparativamente a 2005. Entretanto, a estrutura de distribuição não sofreu impacto, tendo as regióes Norte e Nordeste o maior deficit no acesso a esses serviços comparativamente à região Centro-Sul.

\section{ESTRATÉGIA EMPÍRICA}

Esta seção subdivide-se em duas partes. Na primeira é apresentada a metodologia utilizada para avaliar o impacto da Lei no 11.445/2007 sobre os índices de acesso aos serviços de saneamento ligados ao abastecimento de água, à coleta de lixo e ao esgotamento sanitário. A segunda parte traz a descrição da base de dados empregada nas análises.

\subsection{0 modelo de diferenças em diferenças com pareamento por escore de propensão}

O modelo de DD tem inúmeras aplicaçóes, principalmente quando os dados são provenientes de um experimento natural (ou quase experimento), ou seja, que advém de algum evento exógeno e altera o ambiente (Wooldridge, 2011).

Para avaliarmos as diferenças entre o grupo de tratamento (afetado pela política) e o grupo de controle (não afetado pela política), é necessário o uso de dados antes e depois da política para os dois grupos. Assim, a amostra é dividida em quatro grupos: o grupo de controle antes do evento, o grupo de controle depois do evento, o grupo de tratamento antes do evento e o grupo de tratamento depois do evento.

Como a promulgaçáo da Lei no 11.445 ocorreu em 2007, analisam-se os períodos de 2005 e 2015, por serem períodos anteriores e posteriores à lei, respectivamente. Para definir os grupos de controle e tratamento, utilizou-se o fato de os municípios terem elaborado ou não seus PMSB. Assim, o grupo de tratamento é constituído pelos municípios que, segundo o Panorama dos planos municipais de saneamento básico, haviam elaborado seus PMSB e o grupo de controle é formado pelos municípios que estavam com seus PMSB em elaboração ou inconsistentes.

Uma das dificuldades em utilizar-se o modelo de DD para a avaliação de impacto da Lei do Saneamento Básico é a ausência de um contrafactual adequado para os tratados. O fato é que a efetiva participação dos municípios na lei pode não ser aleatória, o que representa uma fonte de viés que pode advir da ausência de suporte comum entre os grupos de tratamento e controle, devido às características dos grupos não serem sobrepostas (Heckman et al., 1998).

$\mathrm{Na}$ tentativa de eliminar o viés de seleçáo e construir um contrafactual apropriado, utiliza-se a técnica de pareamento por escore de propensão. O objetivo do 
pareamento é encontrar um grupo de controle com características observáveis similares ao grupo de tratamento. No entanto, esta tentativa torna-se cada vez mais difícil quanto maior for a dimensão do vetor de variáveis observáveis $(X)$, e, se colocarmos um número insuficiente de $X$, o pareamento pode não ser eficiente (Pinto, 2012).

Uma forma de contornar esse problema é mediante a criação da função de escore de propensão, proposta por Rosenbaum e Rubin (1983). Assim, em vez de parear os indivíduos com base em todas as características de um determinado vetor $X$, estima-se uma função probabilidade de receber o tratamento baseada em $X$. A função de escore de propensão é entâo definida como:

$$
P(X)=\operatorname{Pr}(T=1 \mid X) .
$$

$\mathrm{O}$ vetor $X^{s}$ inclui características demográficas, socioeconômicas, institucional e geográfica dos municípios brasileiros, a saber: população; índice de desenvolvimento municipal; uma dummy indicando se o município participa ou não de consórcio municipal na área do saneamento e/ou manejo de resíduos sólidos; uma dummy indicando se os municípios pertencem à região geoeconômica Centro-Sul do Brasil; e o número de engenheiros ambientais e civis per capita.

Para que o efeito médio do tratamento sobre os tratados (ATT) seja identificado, duas hipóteses precisam ser satisfeitas.

H1: $\left(Y_{i}^{0} \perp T \mid P(X)\right)$.

Essa hipótese é conhecida como seleção por observáveis (ignorabilidade). A seleção por observáveis requer que a participação no tratamento, condicional às covariadas, seja independente dos resultados. Como implicação, tem-se que o resultado do indivíduo no grupo de controle é um bom previsor do potencial resultado do indivíduo na ausência do tratamento (Pinto, 2012).

A segunda hipótese é a de suporte comum, que define que, para cada indivíduo tratado, exista um outro no grupo de controle com um vetor $X$ similar, condicional ao escore de propensão.

H2: $P(\mathrm{~T}=1 \mid X)<1$.

Neste trabalho, o escore de propensão foi estimado a partir de um Probit. Optou-se pela aplicação de Kernel, ${ }^{10}$ em que todos os tratados são combinados com uma média ponderada de todos os controles a partir de pesos inversamente proporcionais à distância entre os escores de propensão de tratados e controles (Becker e Ichino, 2002).

9. Para mais detalhes das variáveis, consultar a subseção 3.2, que descreve a base de dados.

10. Os testes de pareamento pelo método de vizinhos mais próximos e com uma largura da banda mais restritiva para a função Kernel (em 0,01) foram igualmente satisfatórios. Assim, optou-se pela apresentação dos resultados da aplicação de Kernel com o default da banda (em 0,06), com o uso do comando diff (Stata 15.0), adequado para painel balanceado. 
Após realizar o pareamento, estima-se a regressão com o modelo de DD ponderando-a com os pesos estimados pelo escore de propensão, de forma a contornar o problema da ausência de suporte comum entre o grupo de tratamento e controle. Também se faz uso de um vetor de covariadas $(Z)$ na regressão que contribui para explicar o acesso aos serviços de saneamento básico.

Esse vetor $Z^{11}$ de covariadas é composto pelas variáveis produto interno bruto (PIB) per capita, energia elétrica, população e despesas municipais com saneamento básico per capita. Além disso, cada município possui características não observadas próprias invariantes no tempo, que podem ser descritas como a capacidade proativa do município de fazer planejamento, o seu nível de organização, aspectos institucionais, entre outros. Dessa forma, a equação (4) é estimada por efeitos fixos.

$$
Y_{i t}=\beta_{0}+\beta_{1} \text { PMSB }_{t}+\beta_{2} \text { Tempo }_{t}+\beta_{3} P M S B * \text { Tempo }_{t}+\delta_{i t} Z_{i t}+\varepsilon_{i t},
$$

em que $i=1 \ldots, N$, no qual $N$ é o número de municípios; $t=0$ se os municípios estiverem no período inicial (2005) e $t=1$ caso estejam no período final (2015). A variável $Y_{i}$ é o índice de acesso aos serviços de saneamento básico no município $i$. $P M S B$ é a dummy para o tratamento, que assume valor "1" se o município pertence ao grupo de tratamento, ou seja, se o município de fato elaborou seu PMSB, e valor " 0 " se o município pertence ao grupo controle. Tempo é uma dummy que assume valor "0" se estiver no período anterior ao tratamento (ano de 2005) e valor "1" se a observação está no período pós-tratamento (ano de 2015). Tempo* PMSB, por sua vez, é o termo de interação entre as dummies de Tempo e PMSB. E $Z$ é o conjunto de covariadas da regressão. Além dos efeitos fixos não observáveis.

Os coeficientes $\beta_{0}, \beta_{1}, \beta_{2}, \beta_{3}$ e $\delta$ são os parâmetros não conhecidos a serem estimados, e $\varepsilon$ é o termo de erro que se divide em um termo constante no tempo $a_{i}$ e um termo de erro aleatório $v_{i t^{*}}$ O coeficiente $\beta_{0}$ é o intercepto comum às observaçóes; $\beta_{1}$ denota o efeito de estar no grupo de tratamento independente da política; $\beta_{2}$ é a tendência temporal comum aos grupos; $\beta_{3}$, por sua vez, é o parâmetro de DD que avalia o impacto da Lei do Saneamento Básico de 2007, especificamente o efeito médio do tratamento sobre os tratados.

\subsection{Base de dados}

Utilizam-se como variáveis dependentes os índices de saneamento relativos ao abastecimento de água, à coleta de lixo e ao esgotamento sanitário. As variáveis são provenientes do SIAB, disponível no Datasus para os municípios brasileiros, e indicam o percentual de famílias cadastradas na atenção básica com acesso aos serviços de saneamento básico em cada município brasileiro. 
Assim, os índices de saneamento consistem em: i) percentual de famílias com abastecimento de água por rede geral ou poço; ii) percentual de famílias com lixo coletado; e iii) percentual de famílias com rede de esgoto ou fossa. As variáveis são referentes a dezembro de 2005 e dezembro de 2014 . $^{12}$

A escolha dessa base de dados deve-se ao fato de esta possuir dados para vários anos, além de os dados náo serem autoinformados pelos municípios, tal como ocorre em outras bases de dados de saneamento. Os dados do SIAB permitem conhecer as condiçóes sociossanitárias das famílias cadastradas na atenção básica, sendo um retrato da população usuária dos serviços públicos de saúde no Brasil.

$\mathrm{O}$ vetor $X$ de covariadas utilizadas para a estimação do modelo Probit no pareamento constitui-se pelas variáveis população; índice de desenvolvimento municipal da Federação das Indústrias do Estado do Rio de Janeiro - Firjan (IFDM); uma dummy indicando se o município participa ou não de consórcio municipal na área do saneamento e/ou manejo de resíduos sólidos; uma dummy indicando se os municípios pertencem à regiāo geoeconômica Centro-Sul do Brasil; e o número de engenheiros ambientais e civis per capita.

A variável referente ao tamanho da população é disponibilizada pelo IBGE, com estimativas para os anos de 2005 e 2015 . O IFDM engloba os aspectos: emprego e renda e educação e saúde, sendo adotado como indicador de desenvolvimento dos municípios brasileiros, estando disponível para os anos de 2005 e 2015.

A variável de consórcios municipais é uma dummy que assume valor "1" se os municípios possuem consórcios na área do saneamento básico e/ ou manejo de resíduos sólidos e valor "0" se não os possuem. Esta variável é utilizada como uma variável institucional dos municípios brasileiros no que se refere aos serviços de saneamento básico, uma vez que a Lei do Saneamento Básico de 2007 também faz referência à possibilidade de organização regionalizada na prestação desses serviços. Encontra-se esta variável na base Perfil dos Municípios Brasileiros, disponível no IBGE também para os anos de 2005 e 2015.

A partir da base de dados do Panorama dos Planos Municipais de Saneamento Básico, verifica-se que $80 \%$ dos municípios que elaboraram seus PMSB encontram-se na região Centro-Sul do Brasil. Assim, o padrão de distribuição dos municípios que, de fato, elaboraram seus PMSB também é explicado por uma dummy indicativa da regiáo geoeconômica Centro-Sul, a qual assume valor "1" se o município pertence ao Centro-Sul e "0" caso contrário, sendo esta dummy invariante no tempo.

12. Utilizaram-se os dados de dezembro de 2014 como proxy para o ano de 2015, em razão do número superior de missings e do fato de os dados não estarem sistematizados no Datasus para esse ano. Ademais, ressalta-se que os resultados foram testados para dezembro de 2013 e dezembro de 2015, e os resultados obtidos corroboram as análises apresentadas. 
O número de engenheiros ambientais e civis é utilizado como uma proxy da capacidade técnica dos municípios de elaborarem seus PMSB. Esse número foi retirado da base Rais, do Ministério do Trabalho, para os anos de 2005 e 2015, e dividido pela população total de cada município (per capita).

A variável PMSB é uma dummy que assume valor " 1 " se o município de fato elaborou seu PMSB e valor "0" caso contrário. O total de municípios avaliados pelo panorama são 3.903, dos quais 1.692 elaboraram seus PMSB (tabela 1).

Para as regressóes que adotam o modelo de DD, utiliza-se o seguinte conjunto de covariadas (Z): PIB per capita, energia elétrica, população e despesas municipais com saneamento básico per capita.

A variável PIB per capita está disponível no IBGE e é utilizada como uma medida de renda para os municípios para os anos de 2005 e 2015. A variável energia elétrica indica o percentual de famílias cadastradas na atenção básica com acesso à energia elétrica e serve como uma proxy da infraestrutura dos municípios. Esta variável é proveniente dos dados do SIAB para dezembro de 2005 e dezembro de 2014.

A variável população é a mesma utilizada na estimação do modelo Probit. Já a variável que indica as despesas municipais com saneamento básico foi extraída das Finanças do Brasil (Finbra), para os anos de 2005 e 2015, e dividas pela população total dos municípios.

Para o primeiro teste de robustez, na subseção 4.3, utilizam-se as variáveis citadas anteriormente, para o ano de 2000. Porém, as variáveis indicativas da presença de consórcios municipais na área de saneamento básico e das despesas municipais com saneamento básico não estavam disponíveis para esse ano. Além disso, o índice de desenvolvimento municipal para esse ano foi retirado do Atlas do Desenvolvimento Humano no Brasil, elaborado com dados do Censo 2000.

\section{RESULTADOS}

Esta seção está dividida em três partes. A primeira parte apresenta os testes de médias das variáveis empregadas nas análises no período anterior e posterior à aplicação da Lei do Saneamento Básico. A segunda parte traz a estimação do impacto da lei. E a terceira apresenta as análises de robustez dos resultados.

\subsection{Teste de médias}

As tabelas 2 e 3 apresentam o teste de médias para as variáveis analisadas nos períodos pré e pós-tratamento, respectivamente. Observa-se que as médias das variáveis referentes aos índices de acesso aos serviços de saneamento básico (percentual de famílias com abastecimento de água, lixo coletado e esgotamento sanitário) são 
estatisticamente maiores para o grupo de tratamento comparativamente ao grupo de controle nos períodos anterior e posterior ao tratamento.

Com relação às covariadas PIB per capita, energia elétrica, população, índice IFDM, dummy indicativa da região Centro-Sul e despesas com saneamento, essas também são estatisticamente maiores para o grupo de tratamento vis-à-vis ao grupo de controle nos períodos anterior e posterior ao tratamento.

A dummy que indica a presença de consórcios municipais na área de saneamento básico e manejo de resíduos não obteve diferença estatística entre os grupos de tratamento e controle no ano anterior ao tratamento. Já no ano posterior ao tratamento, a média do grupo de controle para esta variável apresenta-se superior à média do grupo de tratamento. A variável que indica o número de engenheiros ambientais e civis per capita não apresenta diferença estatística entre o grupo de tratamento e o de controle em ambos os períodos.

TABELA 2

Teste de média entre os grupos de controle e tratamento anteriormente à aplicação da Lei do Saneamento Básico

\begin{tabular}{|c|c|c|c|}
\hline Variáveis & Controle & Tratamento & Diferença ${ }^{1}$ \\
\hline \multirow{2}{*}{$\begin{array}{l}\text { Percentual de famílias com abastecimento de água por rede } \\
\text { geral, poço ou nascente }\end{array}$} & 92,57 & 96,43 & $-3,86^{* * *}$ \\
\hline & $(0,28)$ & $(0,22)$ & $(0,38)$ \\
\hline \multirow{2}{*}{ Percentual de famílias com lixo coletado } & 56,30 & 68,02 & $-11,72^{* * *}$ \\
\hline & $(0,59)$ & $(0,66)$ & $(0,90)$ \\
\hline \multirow{2}{*}{ Percentual de famílias com rede de esgoto ou fossa } & 79,04 & 87,37 & $-8,33^{* * *}$ \\
\hline & $(0,45)$ & $(0,41)$ & $(0,63)$ \\
\hline \multirow{2}{*}{ PIB per capita } & 6899,33 & 9548,13 & $-2648,80^{* * *}$ \\
\hline & $(176,23)$ & $(215,42)$ & $(275,86)$ \\
\hline \multirow{2}{*}{ Percentual de famílias com energia elétrica } & 85,55 & 92,90 & $-7,35^{* * *}$ \\
\hline & $(0,37)$ & $(0,29)$ & $(0,49)$ \\
\hline \multirow{2}{*}{ População } & 28879,63 & 48509,85 & $19630,22^{* * *}$ \\
\hline & $(3453,65)$ & $(7444,60)$ & $(7613,77)$ \\
\hline \multirow{2}{*}{ Índice Firjan de Desenvolvimento Municipal } & 0,51 & 0.61 & $-0,10 * * *$ \\
\hline & $(0,00)$ & $(0,00)$ & $(0,00)$ \\
\hline \multirow{2}{*}{$\begin{array}{l}\text { Dummy para consórcio de saneamento e/ou de } \\
\text { resíduos sólidos }\end{array}$} & 0,12 & 0,13 & $-0,01$ \\
\hline & $(0,00)$ & $(0,00)$ & $(0,01)$ \\
\hline \multirow{2}{*}{ Dummy para indicar a região geoeconômica Centro-Sul } & 0,49 & 0,81 & $-0,32^{* * *}$ \\
\hline & $(0,01)$ & $(0,00)$ & $(0,01)$ \\
\hline \multirow[t]{2}{*}{ Engenheiros ambientais e civis per capita } & 0,00 & 0,00 & $-0,00$ \\
\hline & $(0,00)$ & $(0,00)$ & $(0,00)$ \\
\hline \multirow[t]{2}{*}{ Despesas com saneamento per capita } & 13,99 & 16,18 & $-2,18^{* *}$ \\
\hline & $(0,58)$ & $(0,78)$ & $(0,95)$ \\
\hline
\end{tabular}

Elaboração dos autores.

Nota: ${ }^{*}$ Significativo a $10 \% ;{ }^{* *}$ significativo a $5 \% ; e^{* * *}$ significativo a $1 \%$.

Obs.: Erros-padrão entre parênteses. 
TABELA 3

Teste de média entre os grupos de controle e tratamento posteriormente à aplicação da Lei do Saneamento Básico

\begin{tabular}{lrrr}
\hline Variáveis & Controle & Tratamento & Diferença' \\
\hline Percentual de famílias com abastecimento de água por & 94,24 & 97,69 & $-3,45^{* * *}$ \\
rede geral, poço ou nascente & $(0,19)$ & $(0,17)$ & $(0,31)$ \\
& 66,50 & 77,28 & $-10,78^{* * *}$ \\
Percentual de famílias com lixo coletado & $(0,39)$ & $(0,54)$ & $(0,70)$ \\
& 86,17 & 92,58 & $-6,41^{* * *}$ \\
Percentual de famílias com rede de esgoto ou fossa & $(0,26)$ & $(0,30)$ & $(0,45)$ \\
& 17075,53 & 25068,36 & $-7992,83^{* * *}$ \\
PIB per capita & $(298,91)$ & $(504,13)$ & $(561,99)$ \\
& 94,52 & 97,38 & $-2,86^{* * *}$ \\
Percentual de famílias com energia elétrica & $(0,14)$ & $(0,12)$ & $(0,24)$ \\
& 29477,08 & 53402,63 & $-23925,55^{* * *}$ \\
População & $(2237,14)$ & $(8016,29)$ & $(6282,70)$ \\
& 0.63 & 0,70 & $0,07^{* * *}$ \\
Índice Firjan de Desenvolvimento Municipal & $(0,00)$ & $(0,00)$ & $(0,00)$ \\
& 0,27 & 0,24 & $0,03^{* * *}$ \\
Dummy para consórcio de saneamento e/ou de & $(0,00)$ & $(0,01)$ & $(0,01)$ \\
resíduos sólidos & 0,45 & 0,81 & $-0,36^{* * *}$ \\
Dummy para indicar a região geoeconômica Centro-Sul & $(0,00)$ & $(0,00)$ & $(0,01)$ \\
Engenheiros ambientais e civis per capita & 0,00 & 0,00 & $-0,00$ \\
Despesas com saneamento per capita & $(0,00)$ & $(0,00)$ & $(0,00)$ \\
& 29,09 & 39,94 & $-10,85^{* * *}$ \\
& $(0,96)$ & $(1,83)$ & $(1,89)$ \\
\hline
\end{tabular}

Elaboração dos autores.

Nota: 1 * Significativo a $10 \%$; * significativo a $5 \%$; e ** significativo a $1 \%$.

Obs.: Erros-padrão entre parênteses.

O teste de médias mostrou que os grupos de tratamento e de controle não são estatisticamente iguais nas características observadas; com isso, parte-se para a verificação do impacto da Lei do Saneamento Básico de 2007.

\subsection{Estimação do modelo de DD com pareamento por escore de propensão}

Como o fato de os municípios terem ou não elaborado seus PMSB pode estar vinculado às características observáveis intrínsecas aos municípios, ou seja, as trajetórias entre os grupos de tratamento e controle podem não ser paralelas na ausência de tratamento, utiliza-se a técnica de pareamento por escore de propensão, a fim de se obter um contrafactual adequado à análise.

O escore de propensão foi estimado por meio de um modelo Probit condicionado às características socioeconômicas, demográficas, institucional e geográfica dos municípios. A tabela 4 traz os resultados do Probit. 
Os resultados apresentados na tabela 4 mostram que a probabilidade de o município ser tratado, ou seja, ter elaborado seu PMSB, é influenciada positivamente pelo valor do seu IFDM e pelo fato de este pertencer à regiáo geoeconômica Centro-Sul.

O IFDM sintetiza os aspectos relativos a emprego e renda e educação e saúde nos municípios. Já a significância estatística da variável Centro-Sul pode ser explicada pelo fato de a maior parte dos municípios que elaboraram seus PMSB encontrarem-se nesta região (tabela 1).

\section{TABELA 4}

Estimação do Probit para o cálculo do escore de propensão

\begin{tabular}{|c|c|c|}
\hline Variáveis & Coeficientes & $P>|z|^{1}$ \\
\hline População & $\begin{array}{c}0,00 \\
(0,00)\end{array}$ & 0,51 \\
\hline Índice Firjan de Desenvolvimento Municipal & $\begin{array}{c}2,67 \\
(0,24)\end{array}$ & $0,00 * * *$ \\
\hline Dummy para consórcio de saneamento e/ou de resíduos sólidos & $\begin{array}{c}0,03 \\
(0,06)\end{array}$ & 0,66 \\
\hline Dummy para indicar a região geoeconômica Centro-Sul & $\begin{array}{c}0,36 \\
(0,06)\end{array}$ & $0,00^{* * *}$ \\
\hline Engenheiros ambientais e civis, per capita & $\begin{array}{c}-6,64 \\
(23,22)\end{array}$ & 0,77 \\
\hline Intercepto & $\begin{array}{l}-1,93 \\
(0,11)\end{array}$ & $0,00 * * *$ \\
\hline Pseudo-R2 & & 0,11 \\
\hline Número de observações & & 3.496 \\
\hline
\end{tabular}

Elaboração dos autores.

Nota: ${ }^{*}$ Significativo a $10 \% ;{ }^{* *}$ significativo a $5 \%$; e *** significativo a $1 \%$.

Obs.: Erros-padrão entre parênteses.

O gráfico 1 apresenta a função de densidade do escore de propensão entre os municípios tratados e não tratados, na qual se observa um bom ajustamento. A diferença entre esses grupos apresenta-se principalmente nas caudas superiores da distribuição. 


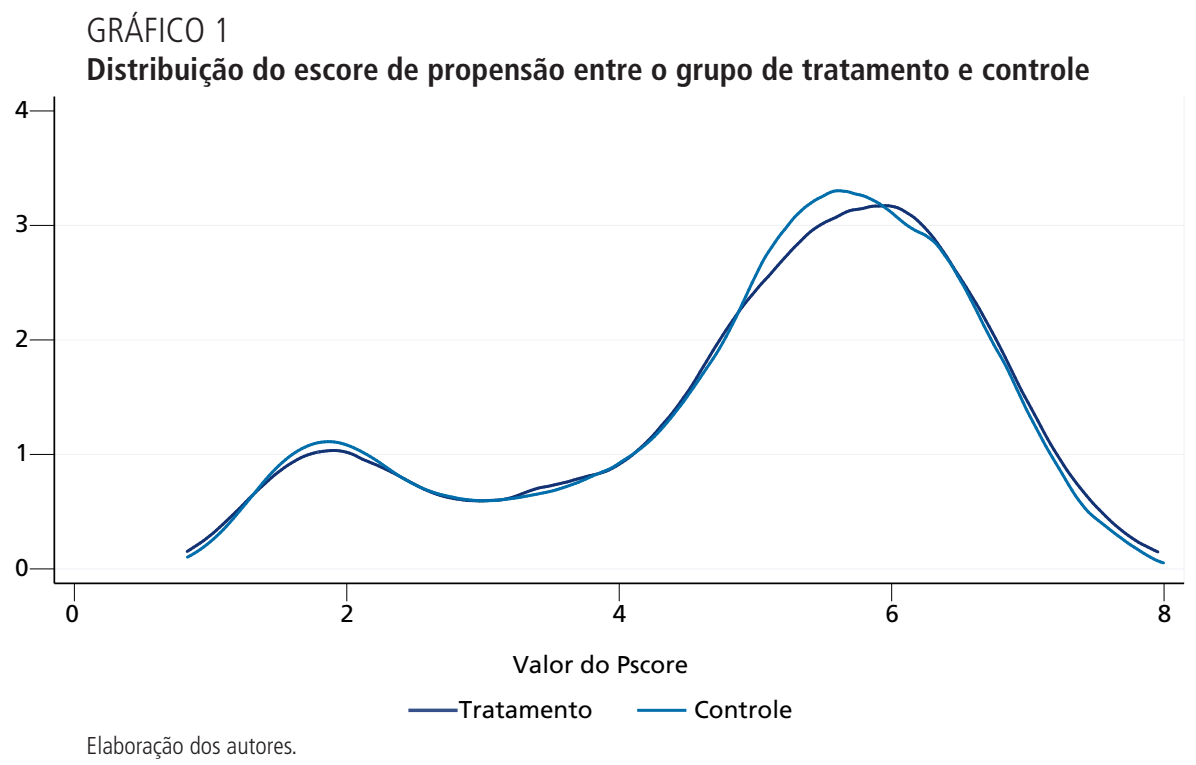

Após a aplicação do pareamento, cabe analisar se, controlando por características observáveis - mediante uso das covariadas PIB per capita, energia elétrica, população e despesas municipais com saneamento básico per capita, além de características não observáveis fixas ${ }^{13}$ no tempo (efeitos fixos) -, a Lei do Saneamento possui impacto sobre os índices de acesso aos serviços de saneamento básico. $\mathrm{O}$ modelo de DD foi calculado na região de suporte comum, e a tabela 5 traz os resultados desta estimação.

Pela tabela 5, observa-se que a Lei do Saneamento Básico possui impacto positivo e significativo nos índices de acesso aos serviços de coleta de lixo e esgotamento sanitário e não significativo para o índice de acesso aos serviços de abastecimento de água. Quanto ao impacto não significativo da lei sobre o índice de acessos aos serviços de abastecimento de água, este se respalda no fato de esse serviço apresentar a maior cobertura comparativamente aos demais serviços de saneamento, uma vez que foi priorizado por outros planos nacionais, como o Planasa (Leoneti et al., 2015).

A covariada energia elétrica possui impacto positivo e significativo para a explicação dos índices de acesso aos serviços de saneamento básico. As variáveis PIB per capita, população e despesas com saneamento básico apresentam impacto próximo de zero, o que pode estar ligado ao fato de essas variáveis serem positivamente correlacionadas ${ }^{14}$ à variável energia elétrica, além de não terem impacto, devido aos demais controles de efeitos fixos existentes na regressão.

13. As estimativas da tabela 5 foram também testadas pelo método de primeiras diferenças, o qual proporcionou resultados similares, uma vez que $\mathrm{T}=2$ (Wooldridge, 2011).

14. Essas correlações são inferiores à 0,27 e não implicam multicolinearidade. 


\begin{tabular}{|c|c|c|c|c|}
\hline & $\begin{array}{c}\text { Percentual de famílias com abas- } \\
\text { tecimento de água por rede geral, } \\
\text { poço ou nascente }{ }^{1}\end{array}$ & $\begin{array}{l}\text { Percentual de famílias } \\
\text { com lixo coletado }{ }^{1}\end{array}$ & $\begin{array}{r}\text { Percentual de famílias con } \\
\text { de esgoto ou fossa' }\end{array}$ & $\begin{array}{l}m \text { rede } \\
1\end{array}$ \\
\hline \multirow[t]{2}{*}{ DD } & 0,28 & $1,32^{* * *}$ & $0,58^{* *}$ & \\
\hline & $(0,18)$ & $(0,39)$ & $(0,27)$ & \\
\hline \multirow[t]{2}{*}{ Tempo } & 0,23 & $6,34^{* * *}$ & $3,36^{* * *}$ & \\
\hline & $(0,17)$ & $(0,36)$ & $(0,25)$ & \\
\hline \multirow[t]{2}{*}{ PIB per capita } & $-0,00^{* * *}$ & $-0,00$ & $-0,00^{* * *}$ & \\
\hline & $(0,00)$ & $(0,00)$ & $(0,00)$ & \\
\hline \multirow[t]{2}{*}{ Energia elétrica } & $0,17^{* * *}$ & $0,34^{* * *}$ & $0,39 * * *$ & \\
\hline & $(0,01)$ & $(0,02)$ & $(0,01)$ & \\
\hline \multirow[t]{2}{*}{ População } & $0,00^{* \star *}$ & $-0,00^{* * *}$ & 0,00 & \\
\hline & $(0,00)$ & $(0,00)$ & $(0,00)$ & \\
\hline \multirow[t]{2}{*}{$\begin{array}{l}\text { Despesas com saneamento } \\
\text { per capita }\end{array}$} & 0,00 & $-0,00$ & $-0,00^{* * *}$ & \\
\hline & $(0,00)$ & $(0,00)$ & $(0,00)$ & \\
\hline \multirow[t]{2}{*}{ Constante } & $79,82^{* * *}$ & $40,87^{* * *}$ & $51,96^{* * *}$ & \\
\hline & $(0,99)$ & $(2,13)$ & $(1,46)$ & \\
\hline R-quadrado: intra= & 0,13 & 0,39 & 0,39 & \\
\hline entre $=$ & 0,07 & 0,02 & 0,33 & \\
\hline total $=$ & 0,07 & 0,03 & 0,32 & \\
\hline Sigma_u= & 9,81 & 25,10 & 14,30 & \\
\hline Sigma_e $=$ & 3,55 & 7,61 & 5,24 & \\
\hline rho $=$ & 0,88 & 0,91 & 0,88 & \\
\hline Número de observações & & & & 6399 \\
\hline Número de grupos & & & & 3459 \\
\hline
\end{tabular}

Elaboração dos autores.

Nota: ${ }^{1 *}$ Significativo a $10 \% ;{ }^{* *}$ significativo a $5 \%$; e *** significativo a $1 \%$.

Obs.: Erros-padrão entre parênteses.

\subsection{Análise de robustez}

Com o objetivo de analisar a robustez das estimativas encontradas pelo modelo de DD no que tange ao impacto da Lei do Saneamento Básico de 2007, foram realizados dois testes. O primeiro teste consiste em examinar os resultados anteriormente à aplicação da política. O segundo teste avalia o resultado ampliando o grupo de controle, incluindo os municípios que náo apresentaram informação no Panorama dos Planos Municipais de Saneamento Básico, ou seja, os quais não se sabe se elaboraram ou não seus PMSB. 
Como a Lei do Saneamento Básico foi promulgada em 2007, utilizam-se os anos de 2000 e 2005 para o primeiro teste de robustez, de forma que o período de tratamento passa a corresponder ao ano de 2005, mantendo-se no grupo de tratamento os municípios que de fato haviam elaborado seus PMSB e no grupo de controle os municípios que estavam com seus PMSB em elaboração ou inconsistentes, segundo informaçóes do panorama.

Como há a possibilidade de ausência de suporte comum entre os municípios de tratamento e controle mesmo no período anterior ao tratamento, utiliza-se a técnica de pareamento por escore de propensão. $\mathrm{O}$ escore de propensão foi estimado por meio de um modelo Probit, utilizando as mesmas variáveis ${ }^{15}$ do modelo apresentado na tabela 4, para os anos de 2000 e 2005.

A estimação do modelo Probit é apresentada na tabela 6, na qual se nota que a probabilidade de o município ter elaborado seu PMSB é positivamente impactada pelo valor do seu índice de desenvolvimento e pelo fato de este pertencer à região geoeconômica Centro-Sul.

TABELA 6

Estimação do modelo Probit para o cálculo do escore de propensão para o primeiro teste de robustez

\begin{tabular}{lcc}
\hline Variáveis & Coeficientes & $P>|z|^{1}$ \\
\hline População & 0,00 & 0,55 \\
& 0,00 & $0,00^{* * *}$ \\
Índice de Desenvolvimento Municipal & 3,08 & $0,00^{* * *}$ \\
& $(0,37)$ & \\
Dummy para indicar a região geoeconômica Centro-Sul & 0,45 & 0,78 \\
& $(0,07)$ & $0,00^{* * *}$ \\
Engenheiros ambientais e civis per capita & $-5,11$ & 0,11 \\
Intercepto & $(18,37)$ & 2.988 \\
Pseudo-R2 & $-2,14$ & $(0,17)$ \\
Número de observações & & \\
\hline
\end{tabular}

Elaboração dos autores.

Nota: ${ }^{*}$ * Significativo a $10 \%$; * significativo a $5 \%$; e ** significativo a $1 \%$.

Obs.: Erros-padrão entre parênteses.

Posteriormente, estima-se a regressão do modelo de $\mathrm{DD}$ com pareamento por escore de propensão. Os resultados estão na tabela 7 , na qual se observa que a simulação do impacto da Lei do Saneamento Básico é não significativa para o índice de abastecimento de água e negativamente significativa para os índices de acesso aos serviços de coleta de lixo e esgoto. Esse impacto negativo reforça os resultados positivos obtidos pela lei após sua efetiva aplicação em 2007.

15. A variável indicativa da presença de consórcios municipais na área de saneamento básico não foi incluída na estimação por não possuir dados disponíveis para o ano de 2000. 
TABELA 7

Resultado da estimação do modelo de DD com pareamento por escore de propensão para o primeiro teste de robustez

\begin{tabular}{|c|c|c|c|c|}
\hline & $\begin{array}{l}\text { Percentual de famílias com abasteci- } \\
\text { mento de água por rede geral, poço } \\
\text { ou nascente }{ }^{1}\end{array}$ & $\begin{array}{l}\text { Percentual de famílias } \\
\text { com lixo coletado }{ }^{1}\end{array}$ & $\begin{array}{l}\text { Percentual de famílias com } \\
\text { esgoto ou fossa }{ }^{1}\end{array}$ & rede de \\
\hline \multirow[t]{2}{*}{$\mathrm{DD}^{\prime}$} & $-0,31$ & $-1,88^{* *}$ & $-0,82^{*}$ & \\
\hline & $(0,30)$ & $(0,75)$ & $(0,44)$ & \\
\hline \multirow[t]{2}{*}{ Tempo } & $64,33^{* * *}$ & $-40,32^{* * *}$ & 9,62 & \\
\hline & $(1,17)$ & $(2,88)$ & $(1,70)$ & \\
\hline \multirow[t]{2}{*}{ PIB per capita } & $0,00^{* * *}$ & $0,00^{* * *}$ & $0,00 * * *$ & \\
\hline & $(0,00)$ & $(0,00)$ & $(0,00)$ & \\
\hline \multirow[t]{2}{*}{ Energia elétrica } & $0,33^{* * *}$ & $1,16^{* * *}$ & $0,83^{* * *}$ & \\
\hline & $(0,01)$ & $(0,03)$ & $(0,02)$ & \\
\hline \multirow[t]{2}{*}{ População } & $-0,00^{* *}$ & $0,00 * * *$ & 0,00 & \\
\hline & $(0,00)$ & $(0,00)$ & $(0,00)$ & \\
\hline \multirow[t]{2}{*}{ Constante } & 0,39 & $-9,62^{* * *}$ & $-1,88^{* * *}$ & \\
\hline & $(0,34)$ & $(0,84)$ & $(0,49)$ & \\
\hline R-quadrado: intra= & 0,99 & 0,92 & 0,98 & \\
\hline entre $=$ & 0,49 & 0,18 & 0,59 & \\
\hline total $=$ & 0,97 & 0,61 & 0,95 & \\
\hline Sigma_u= & 5,78 & 24,44 & 6,89 & \\
\hline Sigma_e= & 5,83 & 14,34 & 8,48 & \\
\hline rho $=$ & 0,49 & 0,74 & 0,39 & \\
\hline Número de observações & & & & 5.904 \\
\hline Número de grupos & & & & 2.972 \\
\hline
\end{tabular}

Elaboração dos autores.

Nota: ${ }^{*}$ Significativo a $10 \%$; ${ }^{* *}$ significativo a $5 \%$; e ** significativo a $1 \%$.

Obs.: Erros-padrão entre parênteses.

Para o segundo teste de robustez, utilizam-se como grupo de controle os municípios que, segundo o panorama, possuíam seus PMSB em elaboração ou inconsistentes, incluindo também os municípios que não possuíam informação no panorama. As estimaçóes são realizadas para os anos de 2005 e 2015. A estimação do Probit é apresentada na tabela 8.

TABELA 8

Estimação do modelo Probit para o cálculo do escore de propensão para o segundo teste de robustez

\begin{tabular}{lcc}
\hline \multicolumn{1}{c}{ Variáveis } & Coeficientes & $P>|z|^{1}$ \\
\hline \multirow{2}{*}{ População } & 1,50 & 0,18 \\
& $(1,12)$ & $0,00^{* * *}$ \\
\hline
\end{tabular}


A Lei do Saneamento Básico e seu Impacto nos Índices de Acesso aos Serviços de

(Continuação)

\begin{tabular}{lcc}
\hline \multicolumn{1}{c}{ Variáveis } & Coeficientes & $P>|z|^{1}$ \\
\hline Dummy para consórcio de saneamento e/ou de resíduos sólidos & 0,02 & 0,67 \\
& $(0,06)$ & $0,00^{* * *}$ \\
Dummy para indicar a região geoeconômica Centro-Sul & 0,44 & 0,75 \\
Engenheiros ambientais e civis, per capita & $(0,06)$ & $0,00^{* * *}$ \\
Intercepto & $-8,28$ & 0.11 \\
Pseudo-R2 & $(26,62)$ & 3.496 \\
Número de observações & $(0.10)$ & \\
\hline
\end{tabular}

Elaboração dos autores.

Nota: ${ }^{*}$ * Significativo a $10 \%$; ${ }^{* *}$ significativo a $5 \%$; e *** significativo a $1 \%$.

Obs.: Erros-padrão entre parênteses.

Pela tabela 9, nota-se que a Lei do Saneamento Básico de 2007 possui impacto positivo e significativo nos índices de acesso aos serviços de coleta de lixo e esgotamento sanitário e não significativo para o índice de acesso aos serviços de abastecimento de água, corroborando a estimação de impacto da lei apresentada pela tabela 5 .

\section{TABELA 9}

Resultado da estimação do modelo de DD com pareamento por escore de propensão para o segundo teste de robustez

\begin{tabular}{|c|c|c|c|}
\hline & $\begin{array}{l}\text { Percentual de famílias com } \\
\text { abastecimento de água por rede } \\
\text { geral, poço ou nascente }{ }^{1}\end{array}$ & $\begin{array}{l}\text { Percentual de famílias com lixo } \\
\text { coletado }{ }^{1}\end{array}$ & $\begin{array}{c}\text { Percentual de famílias } \\
\text { com rede de esgoto ou } \\
\text { fossa }{ }^{1}\end{array}$ \\
\hline \multirow[t]{2}{*}{ DD } & 0,19 & $1,39 * * *$ & $0,44^{* *}$ \\
\hline & $(0,14)$ & $(0,33)$ & $(0,23)$ \\
\hline \multirow[t]{2}{*}{ Tempo } & $0,35^{* * *}$ & $5,84^{* * *}$ & $3,19 * * *$ \\
\hline & $(0,13)$ & $(0,30)$ & $(0,21)$ \\
\hline \multirow[t]{2}{*}{ PIB per capita } & $-0,00 * * *$ & 0,00 & $-0,00 * * *$ \\
\hline & $(0,00)$ & $(0,00)$ & $(0,00)$ \\
\hline \multirow[t]{2}{*}{ Energia elétrica } & $0,17^{* * *}$ & $0,36^{* * *}$ & $0,42^{* * *}$ \\
\hline & $(0,00)$ & $(0,02)$ & $(0,01)$ \\
\hline \multirow[t]{2}{*}{ População } & $0,00^{* * *}$ & $-0,00 * * *$ & 0,00 \\
\hline & $(0,00)$ & $(0,00)$ & $(0,00)$ \\
\hline \multirow[t]{2}{*}{$\begin{array}{l}\text { Despesas com saneamen- } \\
\text { to per capita }\end{array}$} & $-0,00$ & $-0,01$ & $-0,00 * * *$ \\
\hline & $(0,00)$ & $(0,00)$ & $(0,00)$ \\
\hline \multirow[t]{2}{*}{ Constante } & $80,27^{* * *}$ & $38,70^{* * *}$ & $49,19 * * *$ \\
\hline & $(0,69)$ & $(1,87)$ & $(1,31)$ \\
\hline R-quadrado: intra= & 0,14 & 0,39 & 0,39 \\
\hline
\end{tabular}




\begin{tabular}{lccc} 
(Continuação) & & \\
\hline & $\begin{array}{c}\text { Percentual de famílias com } \\
\text { abastecimento de água por rede } \\
\text { geral, poço ou nascente }\end{array}$ & $\begin{array}{c}\text { Percentual de famílias com lixo } \\
\text { coletado }\end{array}$ & $\begin{array}{c}\text { Percentual de famílias } \\
\text { com rede de esgoto ou } \\
\text { fossa' }^{1}\end{array}$ \\
\hline entre= & 0,09 & 0,05 & 0,41 \\
total= & 0,09 & 0,07 & 0,39 \\
Sigma_u= & 10,58 & 24,12 & 14,32 \\
Sigma_e= & 3,23 & 7,61 & 5,31 \\
rho= & 0,91 & 0,91 & 0,88 \\
Número de observações & 9.136 & 9.136 & 9.136 \\
Número de grupos & 4.960 & 4.960 & 4.960 \\
\hline
\end{tabular}

Elaboração dos autores.

Nota: ${ }^{*}$ Significativo a $10 \% ;{ }^{* *}$ significativo a $5 \%$; $e^{* * *}$ significativo a $1 \%$.

Obs.: Erros-padrão entre parênteses.

\section{CONCLUSÕES}

Este trabalho teve como objetivo avaliar o impacto da Lei no $11.445 / 2007$, Lei de Saneamento Básico de 2007, nos índices de acesso aos serviços de abastecimento de água, coleta de lixo e esgotamento sanitário dos municípios brasileiros.

A estimativa do impacto da lei foi realizada por meio do modelo de DD com pareamento por escore de propensão, utilizando como grupo de tratamento o fato de os municípios terem cumprido a Lei oㅡ 11.445/2007, mediante a elaboração do plano municipal de saneamento básico. As estimaçóes também foram controladas para características dos municípios invariantes no tempo, pela aplicação do modelo de efeitos fixos.

A partir dos resultados do pareamento, observa-se que o fato de os municípios terem elaborado seus PMSB é positivamente impactado pelo valor de seus índices de desenvolvimento e pelo fato de estes pertencerem à região geoeconômica Centro-Sul do Brasil.

Já a aplicação e o cumprimento da lei por parte dos municípios demonstra ter impacto positivo e significativo nos índices de coleta de lixo e esgotamento sanitário dos municípios brasileiros e não significativo para o índice de acesso aos serviços de abastecimento de água.

De forma geral, os resultados apontam que as melhorias do acesso aos serviços de saneamento básico no Brasil ao longo dos anos 2000 estáo vinculadas às melhorias das condiçóes socioeconômicas como um todo e pelo instrumento institucional consonante à aplicação da Lei no 11.445/2007.

Entretanto, o baixo número de municípios que, de fato, elaboraram seus PMSB, de acordo com o Panorama dos Planos Municipais de Saneamento Básico, chama atenção para a precariedade do planejamento público no Brasil. 
Apesar disso, espera-se que a Lei do Saneamento Básico de 2007 represente efetivamente um instrumento para a universalização do acesso da população aos serviços de saneamento básico, uma vez que estes serviços são essenciais à prevenção de doenças e agravos à saúde.

\section{REFERÊNCIAS}

AFONSO, D. L.; PEROBELLI, F. S. Oferta pública dos serviços de saúde: uma análise para os municípios brasileiros (2007-2014). Revista de Desenvolvimento Econômico, v. 2, p. 212-234, 2018.

ATLAS DO DESENVOLVIMENTO HUMANO NO BRASIL. Disponível em: $<$ http://atlasbrasil.org.br/2013/pt/o_atlas/o_atlas_/>. Acesso em: 13 fev. 2019.

BECKER, S. O.; ICHINO, A. Estimation of average treatment effects based on propensity score. Stata Journal, v. 2, n. 4, p. 358-377, 2002. Disponível em: <https://bit.ly/3g5sHZ0>. Acesso em: 18 jan. 2018.

BRASIL. Lei no 11.445 , de 5 de janeiro de 2007. Estabelece as diretrizes nacionais para o saneamento básico; cria o Comitê Interministerial de Saneamento Básico; altera as Leis nos 6.766, de 19 de dezembro de 1979, 8.666, de 21 de junho de 1993, e 8.987, de 13 de fevereiro de 1995; e revoga a Lei no 6.528, de 11 de maio de 1978. Brasília: Congresso Nacional, 2007.

. Conceitos, características e interfaces dos serviços públicos de saneamento básico. Brasília: MCidades, 2009. (Lei Nacional de Saneamento Básico: perspectivas para as políticas e gestão dos serviços públicos, v. 2).

. Decreto no 7.217, de 21 de junho de 2010. Regulamenta a Lei no 11.445 , de 5 de janeiro de 2007, que estabelece diretrizes nacionais para o saneamento básico, e dá outras providências. Brasília: Presidência da República, 2010.

. Plano Nacional de Saneamento Básico (Plansab). Brasília: MCidades, 2013. Disponível em: <https://bit.ly/2JIM0vt>. Acesso em: 15 jan. 2018. . PAC atendeu 'uma Inglaterra' com saneamento básico em oito anos. Portal Saneamento Brasil, 12 fev. 2016. Disponível em: <https://bit.ly/36Rx8Ur>. Acesso em: 13 abr. 2018.

. Panorama dos planos municipais de saneamento básico. Brasília: MCidades; Interáguas, 2017. Disponível em: <https://bit.ly/3lMgZnm>.

CARLOS, E. Saneamento: agora vai? Trata Brasil, 2013. Disponível em: <https:// bit.ly/3qCwzWo>. Acesso em: 19 fev. 2019.

DATASUS. Disponível em: <http://datasus.saude.gov.br/>. 
FINBRA - FINANÇAS DO BRASIL. Disponível em: <http://www.tesouro. fazenda.gov.br/contas-anuais>. Acesso em: 20 jan. 2019.

FRISCHTAK, C. R. O investimento em infraestrutura no Brasil: histórico recente e perspectivas. Pesquisa e Planejamento Econômico, v. 38, n. 2, 2008.

HECKMAN, J. et al. Characterizing selection bias using experimental data. Econometrica, v. 66, n. 5, p. 1017-1098, 1998.

HELLER, L. Relação entre saúde e saneamento na perspectiva do desenvolvimento. Ciência e Saúde Coletiva, Rio de Janeiro, v. 3, n. 2, p. 73-84, 1998.

IBGE - INSTITUTO BRASILEIRO DE GEOGRAFIA E ESTATÍSTICA. Disponível em: <https://www.ibge.gov.br/>. Acesso em: 3 mar. 2018.

IFDM - ÍNDICE FIRJAN DE DESENVOLVIMENTO MUNICIPAL. Disponível em: <https://www.firjan.com.br/ifdm/>. Acesso em: 3 de março de 2018.

LEONETI, A. B.; PRADO, E. L.; OLIVEIRA, S. V. W. B. Saneamento básico no Brasil: consideraçóes sobre investimentos e sustentabilidade para o século XXI. Revista de Administraçáo Pública, Rio de Janeiro, v. 45, n. 2, p. 331-348, 2011.

LEONETI, A. B. et al. A parceria público-privada no contexto da universalização do saneamento no Brasil. Desenvolvimento em Questáo, v. 13, n. 32, p. 78-102, 2015.

LISBOA, S. S.; HELLER, L.; SILVEIRA, R. B. Desafios do planejamento municipal de saneamento básico em municípios de pequeno porte: a percepção dos gestores. Engenharia Sanitária e Ambiental, v. 18, n. 4, 2013.

MARCELINI, L. PAC Saneamento: um balanço do programa nas maiores cidades - 2009 a 2015. São Paulo: Trata Brasil, ago. 2016. Disponível em: <https:// bit.ly/3ox5D8s>. Acesso em: 15 jan. 2018.

MASO, R. A. D. Saneamento básico no Brasil: a política nacional entre 1995 e 2007. Porto Alegre: Secretaria do Planejamento, Gestão e Participação Cidadã, 2012. (Texto para Discussão FEE, n. 105).

OMS - ORGANIZAÇÃO MUNDIAL DA SAÚDE. Expert committe on environmental sanitation. Geneva, May 1950.

PEREIRA, T. S. T.; HELLER, L. Planos municipais de saneamento básico: avaliação de 18 casos brasileiros. Engenharia Sanitária e Ambiental, v. 20, n. 3, p. 395-404, 2015.

PINTO, C. C. Pareamento. In: MENEZES FILHO, N. (Org.). Avaliação econômica de projetos sociais. São Paulo: Dinâmica Gráfica e Editora Ltda., 2012.

PIRES, I. M. Planasa: avaliação dos resultados e perspectivas. Revista DAE, São Paulo, v. 121, n. 1212, 1979. 
RAIS. Relaçóes Anuais de Informaçóes Sociais. Disponível em: <http://trabalho. gov.br/rais>. Acesso em 23 de janeiro de 2019.

ROSENBAUM, P. R.; RUBIN, D. B. The central role of the propensity score in observational studies for causal effects. Biometrika, v. 70, n. 1, p. 41-55, 1983. Disponível em: <https://bit.ly/37JWxhU>. Acesso em: 17 jan. 2018.

SAIANI, C. C. S. Deficit de acesso aos serviços de saneamento básico no Brasil Prêmio Ipea-Caixa 2006. Brasília: Ipea, 2006.

SAIANI, C. C. S.; TONETO JÚNIOR, R. Evolução do acesso a serviços de saneamento básico no Brasil (1970 a 2004). Economia e Sociedade, Campinas, v. 19, n. 38, p. 79-106, 2010.

SILVEIRA, A. B. G. Explorando o deficit em saneamento no Brasil: evidências da disparidade urbano-rural. Paranoá, Brasília, n. 10, p. 37-48, 2013.

TRATA BRASIL. Um ano de acompanhamento do PAC Saneamento - de olho no PAC. São Paulo: Instituto Trata Brasil, fev. 2010. Disponível em: <https://bit.ly/38ItFa>. VENSON, A. H.; RODRIGUES, K. C. T. T.; CAMARA, M. R. G. Distribuição espacial do acesso aos serviços de saneamento básico nas microrregióes brasileiras de 2006 a 2013. In: ENCONTRO NACIONAL DA ASSOCIAÇĀO BRASILEIRA DE ESTUDOS REGIONAIS E URBANOS, 13., 2015, Curitiba, Paraná. Anais... São Paulo: Aber; UFPR, 2015.

WOOLDRIDGE, J. M. Introduçáo à econometria: uma abordagem moderna. São Paulo: Thomson, 2011.

Data da submissão: $16 / 4 / 2018$

Primeira decisão editorial em: 11/9/2018

Última versão recebida em: 9/5/2019

Aprovação final em: 22/5/2019 
